\title{
Determination Of The Characteristics Of Polymer-Containing Electronic Elements Using A Mobile Device
}

\section{Sharibayev Nosirjon Yusufjanovich ${ }^{1}$, Ergashev Abdurasul Qodirjonovich ${ }^{2}$, Qaxxarov Maxmudjon Mamadjanovich ${ }^{2}$, Qaxxarov Sobirjon Siddiqjonovich ${ }^{2}$}

\author{
${ }^{1}$ Doctor of Physical and Mathematical Sciences (DSc) of Namangan Engineering-Technological Institute, \\ Namangan, Uzbekistan. \\ E-mail: sharibayev_niti@mail.ru \\ ${ }^{2}$ Candidate of Physical and Mathematical Sciences (PhD) of Namangan Engineering-Technological Institute, \\ Namangan, Uzbekistan. \\ E-mail: abdurasul.ergashev.85@mail.ru
}

Article History: Received:11 January 2021; Accepted: 27 February 2021; Published online: 5 April 2021

\begin{abstract}
In solar energy, which is one of the main solutions to the problems of alternative energy, the use of low-power solar energy and experiments on sensitive photocells have been planned and improved to create a mobile device that increases efficiency and speed of work.
\end{abstract}

Key words: Photo element, Volt Ampere characteristic, mobile device, oscilloscope, organic dye, polymer, semiconductor, channel, signal, sinusoidal, filter, rectifier, temperature, ultrasound, deformation

\section{Introduction}

One of the urgent problems of today, energy shortages and the growing demand for alternative and renewable energy sources is growing rapidly. In this area, the use of solar energy is becoming more efficient. Other types of energy, such as wind, coal, gas, peat, and water potential, are also solar energy, and most of these are considered as economically non-renewable energy. Energy from solar collectors and photovoltaic cells is one of the most important areas for the development of the energy sector today, as it is renewable, environmentally friendly and economically inexpensive and these factors make the area of energetic be urgently developed. Research on the growing demand for solar photovoltaic cells and the reduction of its body cost is leading to the creation of cheaper and more efficient types of semiconductor materials. Metalizationof solar batteries upgrades efficiency of the batteries and, it is a simple and cheap method.[1]In biochemistry laboratories oscilloscopes are widely used.[2]In particular, today it is known to science that the production of polymer composite materials is somewhat convenient and can serve as a light-sensitive energy source. Since polymeric materials are composed of many compounds in terms of structure, the physical processes that take place in them must also be evaluated on the basis of the composition of the compound, and therefore we always need methods to effectively determine the resistance of the compound to various physical influences. Today, polyacrylonitrile-based polymers, ethylene carbonate and propylene carbonate plasticizers, and polymer electrolytes with various amounts of tetrabutylomonium iodide salt and iodine have been developed and used in paints around the world. including sensitive solar cells. The maximum room temperature is obtained for electrolytes with sufficient conductivity. The maximum efficiency of the concentration of a photo element from a photon to an electric energy state is $54.01 \%$. Electrical impedance spectroscopy of the same element shows the lowest series resistance, indicating the superiority of the electrolyte charge transport properties in the DSSS. In addition, the electron transfer time was maintained and the electron recombination time $\mathrm{t}$ rek, charge accumulation efficiency tarqal, electron scattering coefficient D and DSSS length with prepared GPE were calculated by intensity modulated photocurrent spectroscopy and intensity modulated photovolt spectroscopy methods prepared by LP. Electrical impedance spectroscopy of the same element shows the lowest series resistance, indicating the superiority of the electrolyte charge transport properties in the DSSS. In addition, the electron transfer time constant and electron recombination time $\mathrm{t}$ rek, charge collection efficiency $\bar{e}$, electron scattering coefficient D and DSSS length with prepared GPE were calculated by intensity modulated photocurrent spectroscopy and intensity modulated photovolt spectroscopy methods prepared by LP.[3] Preparation of gel polymer paint and obtaining photogalvanic characteristics of its physical quantities determine the resistance of the prepared photocell to the physical impacts occurring during the work process by conducting spectroscopic observations of chemical impedances. [3][4]

\section{Materials}

Since this article is an observation of experimental work, an oscilloscope was used, and for comparison, a voltmeter and perometer, wattmeter, current source, sinusoidal and non-harmonic vibration generators, an ultrasonic generator, and mechanisms representing several types of mechanical deformation were used. The object was observed by using 3 DD207 98.8 transistors, ED 24250180 diode, 13001S8D transistors were observed at the object under study. 
Thehelp of the oscilloscope is important in this area because the basic characteristics of an oscilloscope are determined by the sampling rates and the level of accuracy (quantity levels) of the converter. Once selected, the values are stored in digital memory for later processing and visualization. Digital oscilloscopes can perform complex calculations on the information stored in the memory with the advantage of digital logic. This means that the capability of modern digital oscilloscopes is a big advantage over analog ones.[5]

\section{Methods}

Examples of a polymer-based solar photovoltaic preparation method are liquid electrolytes(LE) and gel polymer electrolytes (GPE). The permeability of these substances is measured after they are prepared. It is possible to conclude about the permeability of a compound obtained at new different quantities with the template obtained by experimentsby comparing. The study of permeability is important in medicine, including physiotherapy. [6] Of course, the permeability also depends on the conditions. [7]It seems very simple to classify chemicals according to their electrical resistance p. For metals it is $10^{-6} \div 10^{-4} \mathrm{OmSm}$. Semiconductors, on the other hand, have a specific resistance in the $10^{-4} \div 10^{10} \mathrm{OmSm}$ range. Substances above $10^{10} \mathrm{OmSm}$ are dielectric. As can be seen from the above examples, the specific resistance values during the transition from one class of substances to another are consistent with each other.[8] Therefore, the specific resistance values cannot serve as a clear criterion for classifying substances. [9]

$$
\rho=\rho_{0}(1+\alpha t)
$$

(1) It can be understood from the expression that an increase in temperature causes an increase in resistance. In formula (1) $\rho_{0}$ - the specific resistance of this metal at $0{ }^{\circ} \mathrm{C}$. Thetemperature dependence coefficient of the $\alpha$ resistance is $1 / 273$. In semiconductors, the nature of the resistance and the temperature dependence of the conductivity are different, for a given temperature range they are determined by the interdependence of the form.

$$
\rho=\rho_{0} e^{\frac{\beta}{t}}
$$

Or permeability

$$
\sigma=\sigma_{0} e^{-\frac{\beta}{t}}
$$

$\rho_{0}$ and $\sigma_{0}$ are constant in formulas (2) and (3),appropriate for a specific temperature range specific to each semiconductor. If a large number of such intervals are observed, the permeability indicates the purity of the observed element, large amount of foreign matter entering the compound. From this concept it is important not to err in the amount of percentages in the preparation of the photo element with gel polymer paint. A special table is used for this.

Table 1. To prepare the electrolyte layer

\begin{tabular}{|c|c|c|c|c|c|c|c|c|c|c|c|}
\hline \multirow{2}{*}{\multicolumn{2}{|c|}{$\begin{array}{l}\text { TPAI } \\
\text { g wt\% }\end{array}$}} & \multicolumn{2}{|c|}{ PEO } & \multicolumn{2}{|c|}{ PC } & \multicolumn{2}{|c|}{ EC } & \multicolumn{2}{|c|}{ DMF } & \multicolumn{2}{|c|}{ I2 } \\
\hline & & \multicolumn{2}{|c|}{$\mathrm{g} w \mathrm{t} \%$} & \multicolumn{2}{|c|}{ g wt $\%$} & \multicolumn{2}{|c|}{ g wt $\%$} & \multicolumn{2}{|c|}{ g wt $\%$} & \multicolumn{2}{|c|}{$\mathrm{g} \mathrm{wt} \%$} \\
\hline 0.05 & 4.33 & 0.1 & 8.65 & 0.25 & 21.65 & 0.25 & 21.95 & 0.5 & 43.29 & 0.005 & 0.43 \\
\hline 0.10 & 8.27 & 0.1 & 8.26 & 0.25 & 0.66 & 0.25 & 20.66 & 0.5 & 41.32 & 0.010 & 0.83 \\
\hline 0.15 & 11.86 & 0.1 & 7.91 & 0.25 & 19.76 & 0.25 & 19.76 & 0.5 & 39.53 & 0.015 & 1.18 \\
\hline 0.2 & 15.15 & 0.1 & 7.58 & 0.25 & 18.94 & 0.25 & 18.94 & 0.5 & 37.88 & 0.020 & 1.52 \\
\hline 0.25 & 18.18 & 0.1 & 7.27 & 0.25 & 18.18 & 0.25 & 18.18 & 0.5 & 36.37 & 0.025 & 1.82 \\
\hline
\end{tabular}

\begin{tabular}{|cccccccccc|}
\hline \multicolumn{2}{|c}{ TPAI } & \multicolumn{2}{c}{ PC } & \multicolumn{2}{c}{ EC } & \multicolumn{2}{c}{ DMF } & \multicolumn{2}{c|}{ I2 } \\
\hline \multicolumn{2}{|c|}{$\mathrm{g} \mathrm{wt} \%$} & \multicolumn{2}{c}{$\mathrm{g} \mathrm{wt} \%$} & \multicolumn{2}{c|}{$\mathrm{g} \mathrm{wt} \%$} & \multicolumn{2}{c|}{$\mathrm{g} \mathrm{wt} \%$} & \multicolumn{2}{c|}{$\mathrm{g} \mathrm{wt} \%$} \\
\hline 0.05 & 4.74 & 0.25 & 23.7 & 0.25 & 23.7 & 0.5 & 47.4 & 0.005 & 0.47 \\
\hline 0.10 & 9.01 & 0.25 & 22.52 & 0.25 & 22.52 & 0.5 & 45.05 & 0.010 & 0.90 \\
\hline 0.15 & 1.87 & 0.25 & 21.46 & 0.25 & 21.46 & 0.5 & 42.92 & 0.015 & 1.29 \\
\hline 0.2 & 16.39 & 0.25 & 20.49 & 0.25 & 20.49 & 0.5 & 40.99 & 0.020 & 1.64 \\
\hline 0.25 & 19.61 & 0.25 & 19.61 & 0.25 & 19.61 & 0.5 & 39.21 & 0.025 & 1.96 \\
\hline
\end{tabular}

Table 2. To prepare the electrolyte layer 
The resulting electrolytes are used to prepare the photocell. The advantage of a photocell with this composition is its high sensitivity.[4][10]The difference between semiconductors and metals is solved by the sign of the temperature coefficient of electrical conductivity. However, selecting it as a determinant complicates the matter by the fact that it can behave like a semiconductor metal over a certain temperature range.[11]Therefore, it is incorrect to say that a substance belongs to the class of semiconductors or metals, depending on its conductivity. A decrease in temperature increases the specific conductivity of metals, which means that there is always the presence of free electrons in the metal, regardless of temperature. In semiconductors, by contrast, the conductivity decreases with decreasing temperature. It follows that free carriers (electrons and cavities) in a semiconductor are formed as a result of thermal motion.[12]Experiments show that the formation of free charge carriers in a semiconductor also occurs under light amd nuclear radiation. Using the resulting conductivity at the expense of the resulting charge carriers, light-sensitive devices and devices for measuring the amount of radioactive radiation are made.[13]The formation of both equilibrium and unbalanced charge carriers depends in many respects on the degree of purity in the structure of the semiconductor material. This means that semiconductors are materials that have a certain conductivity at room temperature, in the range of $10^{-6} \div 10^{4}(\mathrm{Om}-1 \mathrm{Sm}-1)$, which in many respects depends on the structure of the substance, the type and amount of foreign matter, and external conditions.[13] [12] Conductivity depends on external influences such as temperature, light, electric and magnetic fields, and radiation with nuclear particles. For example, the physical processes that take place in a semiconductor in high-frequency oscillations are subject to the relevant laws due to the composition of the material.[14]It is known that there are two types of conductivity: electron and ion. Metals have electronic conductivity.

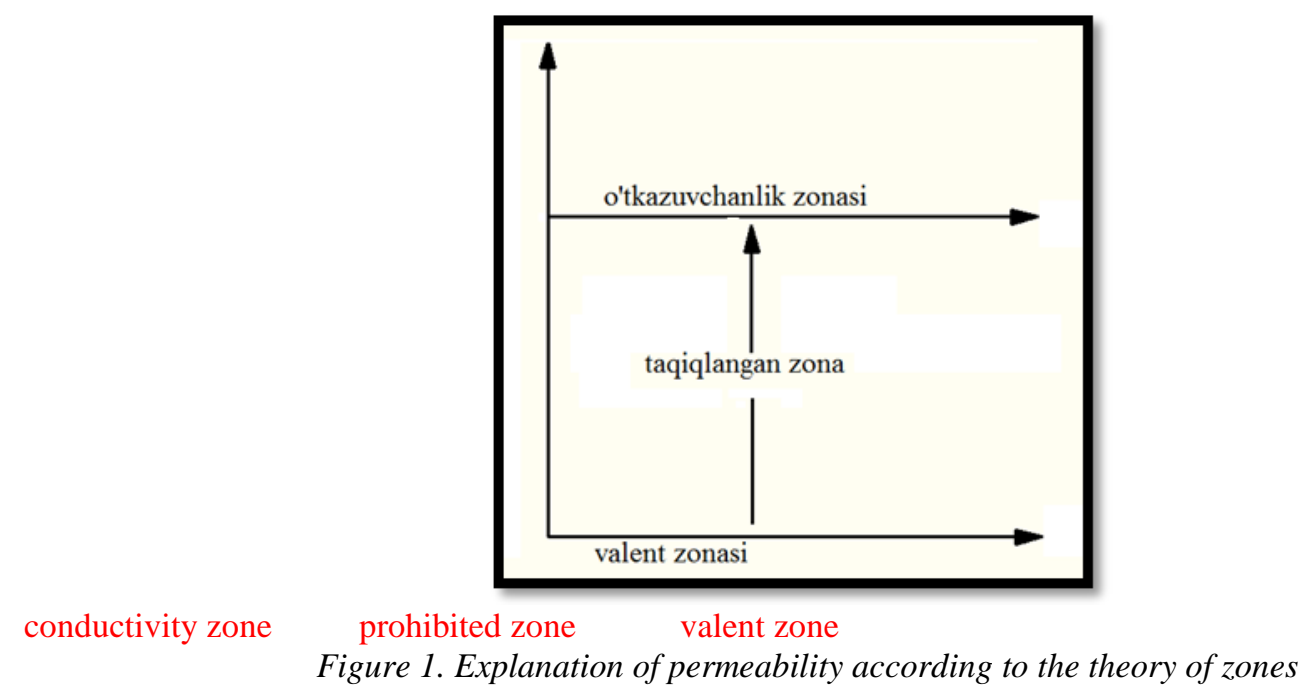

In ionic conductivity, current is passed through the ions of matter. The usual representatives of ionic conductivity are electrolytes. Semiconductors can be both electronic and ionic.[15] In this paper, we consider only the physical properties of electronic semiconductors. Electronic semiconductors contain a large number of different substances. Semiconductors are non-compounding substances: barium B, silicon Si,[16]phosphorus F, sulfur C, germanium Ge, arsenide As, gray tin Sn, selenium Se, tellurium Te, and many complex chemical compounds can be cited as examples. In addition to inorganic substances, some organic substances also have semiconducting properties, such as hydrocarbons benzene, naphthalene, anthracene, naphthalene, coronene, and others.[17]If we consider three types of conductivity, it is possible to observe all of the ionic electrons and hollow permeabilities in polymer ionic compounds. Therefore, it is necessary to study these permeabilities. According to the theory of zones, the conductivity is $>3 \mathrm{eV}$ for the gap - dielectric, $<3 \mathrm{eV}-\mathrm{for}$ the semiconductor, $0 \mathrm{eV}$ - for the conductor.Conductivity of electron-holes in metals (electrons and in some cases holes) and semiconductors (electrons and or holes); The conductivity of metals is mainly carried out by electrons. In the composition of the compound, the electrons in the metal appear in the form of a cloud. The cavities are an unfilled valence bond that appears as a positive charge equal to the charge of the electron. The second type of conductor: the ionic conductivity in the electrolyte is distinguished by the fact that one electrolyte may contain several charge carriers.[18] For example, amphoteric conductivity, ie ionic and electron conductivity.[19] When different charge carriers are present in a conductor, the concept of "transmission number" is introduced. The transmission number is the ratio of the electricity supplied by this type of carrier to the total electrical energy. $t_{i}=\frac{u_{i}}{\sum u_{i}}$ (Formula 4) The cation is the sum (or mobility) of the ion exchange rate given that the ion exchange rate is equal to the ratio of the velocity of the ion.[20]and is understood by the 
mobility of the anion. It can also be defined as a value that characterizes the share of ion current in the total current:

$$
\begin{aligned}
& t_{i}=\frac{I_{i}}{I}=\frac{I}{\sum I_{i}} \\
& t_{i}^{(\sigma)}=\frac{\sigma_{i}}{\sigma} \\
& \sigma_{i}=U_{i} C_{i} Z_{i} e
\end{aligned}
$$

where $\mathrm{Ui}$ is the displacement or electrochemical mobility of the ion, $\mathrm{Ci}$ is the concentration of these ions, [21] Z is their charge. however, ti and tr (s) are not replaced in the presence of a blocked electrode and are not equal to it. If a certain r-level carrier is blocked, there will be a partial current of this component, so if $\operatorname{tr}=0$ at the same time, $\operatorname{tr}(\mathrm{s}) \neq 0$, because $\mathrm{sr} \neq 0$ is also a function of $\operatorname{tr}(\mathrm{s}) \mathrm{s}$ may differ from the sample size. It is also possible to draw conclusions about the resulting conductivity, taking into account the effect of temperature on the conductivity and other external influences. for this, it is of great importance to determine the element permeability of the object under study (polymer-containing ionic conductivity).[22] Therefore, in order to achieve high efficiency in the work, it is necessary to keep the magnitude of the current in the conduction test with great accuracy, as well as to enter external influences, including temperature, variable electromagnetic field frequency, deformation, into the computer.[23]

$$
\sigma=\sigma_{0} e^{\frac{E_{a k t}}{k t}}
$$

One of the external influences is the temperature dependence, which can be seen here. Conductivity in mixed conductors is determined not only by the presence of ionic defects, but also by the concentration of electrons (holes). But there are theoretical concepts, formulas that describe this concentration. [24]

\section{Results and Discussions}

To determine whether the conductivity of a conductive substance depends on external influences in a mobile device, it is necessary to assemble the chain shown in the following diagram. Before planning and drawing the scheme, the mobile devices that were designed and used in several directions were studied.[25][26]

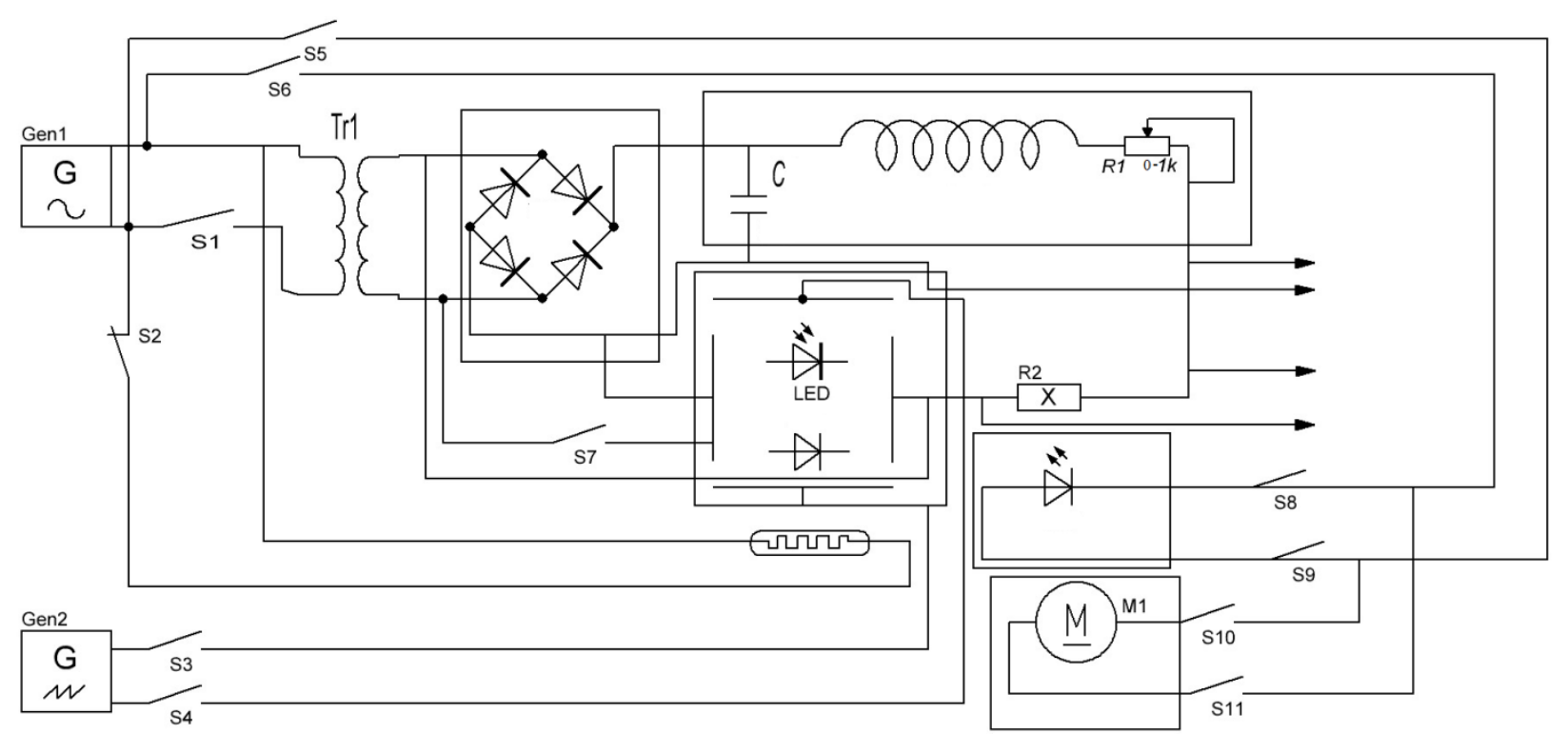

Figure2. the structure of the mobile device in the schematic drawing

This scheme uses a generator that generates sinusoidal and non-sinusoidal electricity (to generate space) and a controlled heat source that provides a temperature 150-200 degrees above room temperature. The device also has a device for mechanical deformation, mechanical vibration and wave transmission, which allows to study the resistance of the conductor to mechanical impacts.[27]

When an alternating current of different frequencies is applied to a conductor, the total resistance varies depending on the frequency Z.[28] From experimental observations and the above considerations, it can be said that in mixed conductors with a polymer content, this total resistance varies, and this is determined by the impedian spectroscopy method.[29] If we use an oscillograph in the device and determine the frequency and send the data to a computer, we will also be able to get a graph of impedances. [30]

The input current from the generator to the system is $220 \mathrm{~V} 50 \mathrm{~Hz}$. The correction factor of transformer 1 should be $4.4 \leq \mathrm{k} \leq 55$. A semiconductor rectifier is used in the rectifier block and the rectified current is filtered. 
To do this, an inductive coil is placed in series in the chain. In parallel, a capacitor is placed. If the voltage is prepared to be controlled in a transformer in the range of $4-50 \mathrm{~V}$, it is controlled at small intervals through a rheostat. The alternating current energy to be supplied to the sample under study passes through several devices until it reaches it, and the energy calculation for this takes place.

$$
\begin{gathered}
W_{\text {tarmoq }}=W_{\text {trs }}+W_{\text {torgrilagich }}+W_{\text {reost }}+W_{\text {obekt }} \\
W=\sum W_{n}
\end{gathered}
$$

Their energy consumption should also be taken into account when conducting experiments with other external influences on the object.[31]

$$
W_{\text {tarmoq }}=W_{\text {trs }}+W_{\text {torgrilagich }}+W_{\text {reost }}+W_{\text {obekt }}+W_{\text {tashqi tasir }}
$$

To channel 2 of the oscilloscope $R_{x}$ if we give the voltage drop of the reference resistance, this channel describes the time $t$ on the $\mathrm{X}$ axis and the value of the voltage drop occurring on the reference resistance at this instantaneous value on the $\mathrm{Y}$ axis. [32]

The signal received in solar photocell is fed to channel 1 of the oscilloscope, where the oscillograph graph shows the voltage of the corresponding solar photocell along the $\mathrm{Y}$ axis at the instantaneous value of time $\mathrm{t}$ on the $\mathrm{X}$ axis. On channel 2

$$
\begin{aligned}
\mathrm{U} 2 & =\mathrm{Push} * \mathrm{Rx} \\
I_{\text {tarmoq }} & =\frac{U_{2}}{R_{X}}
\end{aligned}
$$

that is or in instantaneous values

\begin{tabular}{|c|c|c|c|}
\hline SaveTime: & $\begin{array}{l}2019-12- \\
10 \\
14: 28: 34 \\
\end{array}$ & & \\
\hline \multirow{6}{*}{$\begin{array}{l}\text { Status: } \\
\text { Frequency: } \\
\text { Period: } \\
\text { SP: } \\
\text { PK-PK: }\end{array}$} & & $\mathrm{CH} 1$ & $\mathrm{CH} 2$ \\
\hline & & Trigd & Trigd \\
\hline & & $50,000 \mathrm{~Hz}$ & $49,929 \mathrm{~Hz}$ \\
\hline & & $20,000 \mathrm{mS}$ & $20.029 \mathrm{mS}$ \\
\hline & & $50,000 \mathrm{uS}$ & $50,000 \mathrm{uS}$ \\
\hline & & $190,000 \mathrm{~V}$ & $4,000 \mathrm{~V}$ \\
\hline
\end{tabular}

$$
I_{t n}=\frac{U_{t n}}{R_{X}}
$$

can not be found by expression.

Table 3. A table that appears when exporting data to a computer.

In channel 1 , the $U_{1}$ voltage is plotted on a two column table to plot the points corresponding to a specific time using the solar photocell self-matching voltage. The first column represents the voltage values of solar photocell, while the second column represents the current in it. Using these values, it is possible to draw a Volt Ampere characteristic in any of the graphics programs.[33]

If we have a software tool that draws 3D graphics, we can observe one of the external influences on the 3rd column by expressing one of the temperature, area, frequency, etc.

$$
W_{\text {trs }}=W_{\text {kirish }}-W_{\text {chiqish }}
$$

The energy difference is obtained using a wattmeter. In practice, its power consumption depends on the energy of the consumer, and if the energy of the consumer changes, the energy consumption of the transformer and the rectification block will also change.[34]

\section{Conclusion}

To calibrate a mobile device, it will be possible to obtain a reference rectifier from which the VACh(volt ampere characteristic) has been obtained or previously known, and to retrieve and compare the VACh(volt ampere characteristic)from the device again. If there are nosinusoidal oscillations in the network or if the time intervals are not the same in the channels, the results will not be synchronized and distortions in the graph will occur. The following graph was obtained during the calibration work (Figure 3. and Figure 4.). 


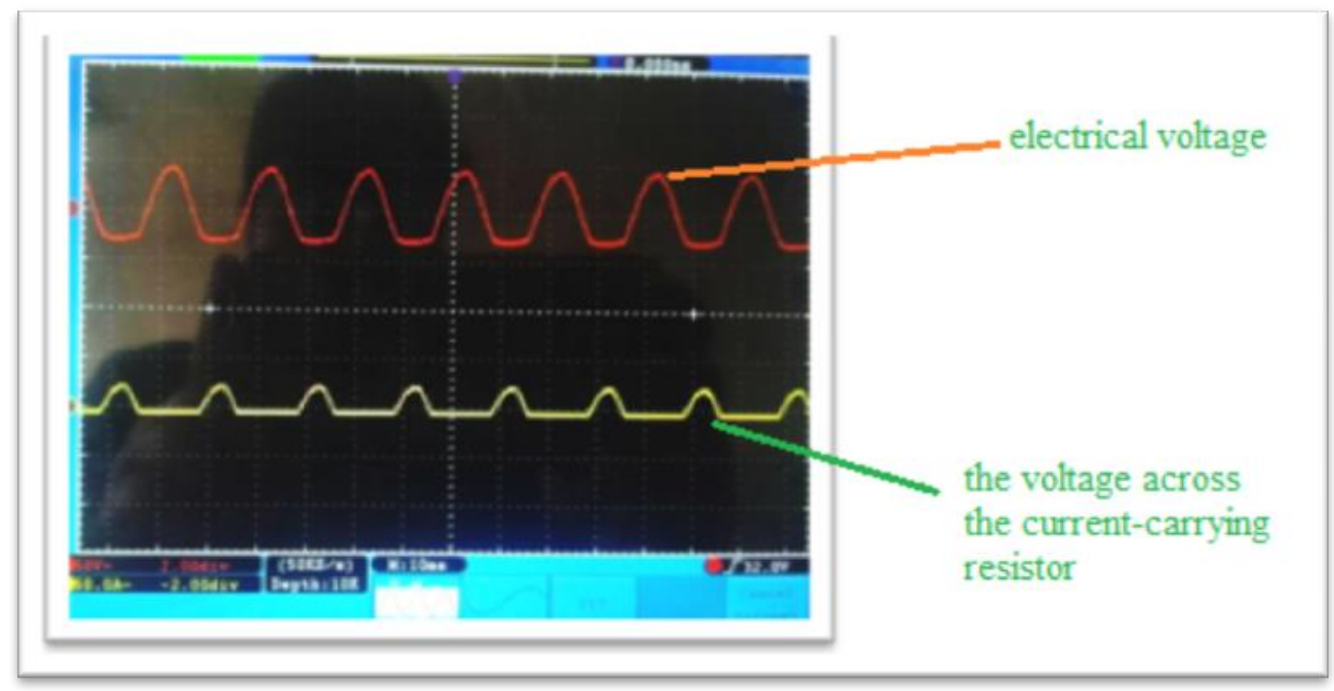

Figure 3. That's rightrecording voltage and current in the connection

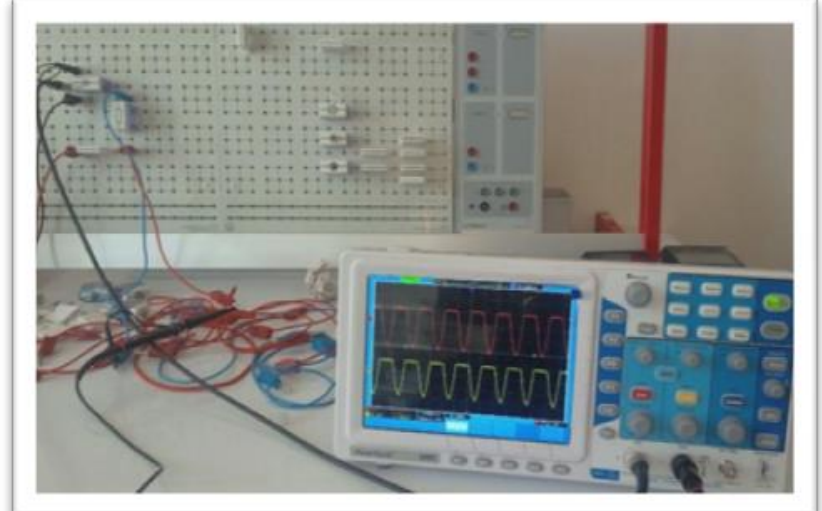

Figure 4.Recording voltage and current in the reverse connection

As can be seen from the graph, the goal is achieved when assembling the device. It is achieved to obtain small time intervals of VACh(volt ampere characteristic)at the object under various external influences. This reduces the errors caused by changes in the physical size of the object under the influence of temperature deviation.

\section{References:}

1. M. Laska and $d r$., «Metallization of solar cells, exciton channel of plasmon photovoltaic effect in perovskite cells», Nano Energy, t. 75, you. 2020, doi: 10.1016 / j.nanoen.2020.104751.

2. Z. Li, L. Liu, Y. Fan, i J. Xi, «Kinetic modeling for high voltage electrical discharge extraction based on discharge energy input», Food Chem., t. 314, June. 2020, doi: 10.1016 / j.foodchem.2020.126168.

3. FI Chowdhury, MH Buraidah, AK Arof, BE Mellander, i IM Noor, "Impact of tetrabutylammonium, iodide and triiodide ions conductivity in polyacrylonitrile based electrolyte on DSSC performance", Sol. Energy, t. 196, ss. 379-388, Jan. 2020, doi: 10.1016 / j.solener.2019.12.033.

4. AK Arof, NA Mat Nor, N. Aziz, MZ Kufian, AA Abdulaziz, i OO Mamatkarimov, «Investigation on morphology of composite poly (ethylene oxide) cellulose nanofibers», v Materials Today: Proceedings, 2019, t. 17, ss. 388-393, doi: 10.1016 / j.matpr.2019.06.265.

5. B. Finish i M. İskefiyeli, «Realization of Digital Oscilloscope with FPGA for Education», Procedia Soc. Behav. Sci., T. 174, ss. 814-820, Feb. 2015, doi: 10.1016 / j.sbspro.2015.01.666.

6. M. Kilicli, M. Baslar, MZ Durak, i O. Sagdic, «Effect of ultrasound and low-intensity electrical current for microbial safety of lettuce», LWT, t. 116, dec. 2019, doi: 10.1016 / j.lwt.2019.108509.

7. XW Yan, M. Joharian, M. Naghiloo, R. Rasuli, ML Hu, i A. Morsali, «Metal - organic framework derived porous 2D semiconductor $\mathrm{C} / \mathrm{ZnO}$ nanocomposite with high electrical conductivity», Mater. Lett., T. 252, ss. 325-328, Oct. 2019, doi: 10.1016/ j.matlet.2019.06.007.

8. F. Semiconductors, «K. V. Shalimova », 1985.

9. SZ Yusof, HJ Woo, MA Careem, i AK Arof, "Gel electrolytes with I- / I3- redox mediator based on methylcellulose for dye-sensitized solar cells”, Opt. Mater. (Amst)., T. 79, ss. 381-389, May 2018, doi: 10.1016 / j.optmat.2018.03.064.

10. G. Gulyamov, NY Sharibaev, and UI Erkaboev, "The Temperature Dependence of the Thermodynamic 
Density of States and Urbach Rule”, World J. Condens. Matter Phys., T. 05, vqp. 01, ss. 43-47, 2015, doi: 10.4236 / wjemp.2015.51006.

11. CW Wu i DX Yao, "Intriguing p-orbital magnetic semiconductors and carrier induced half-metallicity in surface oxygen-functionalized two-dimensional X2N (X = Ca, Sr) crystals", J. Magn. Magn. Mater., T. 493, Jan. 2020, doi: 10.1016 / j.jmmm.2019.165727.

12. G. Gulyamov, AG Gulyamov, AQ Ergashev, i BT Abdulazizov, «THE USE OF PHASE PORTRAITS FOR THE STUDY OF THE GENERATION-RECOMBINATION PROCESSES IN SEMICONDUCTORS».

13. G. Gulyams, MG Dadamirzayev, i NY Sharibaev, «INFLUENCE OF REFLECTION OF PHOTOELECTRONS FROM THE SURFACE PHOTO-EMF IN THIN SEMICONDUCTOR FILMS AND COMPARE IT WITH THE BULK PHOTO-EMF», Altern. Energy Ecol., Vqp. 21, 2016, doi: 10.15518 / isjaee.2015.21.002.

14. G. Gulyamov, MG Dadamirzaev, NY Sharibayev, and N. Zokirov, "EMF of Hot Charge Carriers Arising at the pn-Junction under the Influence of the Microwave Field and Light", J. Electromagn. Anal. Appl., T. 07, vqp. 12, ss. 302-307, 2015, doi: 10.4236/ jemaa.2015.712032.

15. L. Azzouz and dr., «Magnetic semiconductor properties of RbLnSe2 (Ln = Ce, Pr, Nd, Gd): A density functional study», J. Magn. Magn. Mater., T. 501, May 2020, doi: 10.1016 / j.jmmm.2020.166448.

16. 6 Search Results - Authors (AK Arof) - ScienceDirect».

17. SY Yu and $d r$., «Bismuth-containing semiconductors for photoelectrochemical sensing and biosensing», Coordination Chemistry Reviews, t. 393. Elsevier BV, ss. 9-20, 15-Aug-2019, doi: $10.1016 /$ j.ccr.2019.05.008.

18. W. Zhang and $d r$., «The pressureless sintering of micron silver paste for electrical connections», J. Alloys Compd., T. 795, ss. 163-167, July. 2019, doi: 10.1016 / j.jallcom.2019.04.270.

19. EA Moore, AA Mohammed, HM Widatallah, and AI Arbab, «Fluorinated perovskite as magnetic spinpolarized semiconductor», Solid State Commun., t. 294, ss. 39-42, June. 2019, doi: 10.1016 / j.ssc.2019.03.005.

20. Y. Chen, J. Peng, Y. Lai, B. Wu, L. Sun, i J. Weng, "Ultrasensitive label-free detection of circulating tumor cells using conductivity matching of two-dimensional semiconductor with cancer cell", Biosens. Bioelectron., T. 142, Oct. 2019, doi: 10.1016 / j.bios.2019.111520.

21. K. Vijaya Babu i S. Cole, «Luminescence properties of Dy3 + -doped alkali lead alumino borosilicate glasses», Ceram. Int., T. 44, vqp. 8, ss. 9080-9090, June. 2018, doi: 10.1016 / j.ceramint.2018.02.115.

22. F. Bendahma, M. Mana, S. Terkhi, S. Cherid, B. Bestani, i S. Bentata, "Investigation of high figure of merit in semiconductor XHfGe $(\mathrm{X}=\mathrm{Ni}$ and $\mathrm{Pd})$ half-Heusler alloys: Ab-initio study", Comput. Condens. Matter, t. 21, dec. 2019, doi: 10.1016/ j.cocom.2019.e00407.

23. CC Hsu, CC Tsao, YH Chen, i XZ Zhang, "Bipolar resistive switching characteristics of a sol-gel InZnO oxide semiconductor”, Phys. B Condens. Matter, t. 561, ss. 64-69, May 2019, doi: 10.1016 / j.physb.2019.02.048.

24. C. Wang, Y. Wang, Y. Chen, H. Liu, and J. Liu, "User authentication on mobile devices: Approaches, threats and trends," Comput. Networks, t. 170, apr. 2020, doi: 10.1016 / j.comnet.2020.107118.

25. B. Hu, J. Qiu, H. Chen, V. Tao, J. Wang, i H. Lin, "First, second and potential third generation spreads of the COVID-19 epidemic in mainland China: an early exploratory study incorporating location-based service data of mobile devices", Int. J. Infect. Dis., T. 96, ss. 489-495, July. 2020, doi: 10.1016 / j.ijid.2020.05.048.

26. I. Diemberger and $d r$., "The" Subtle "connection between development of cardiac implantable electrical device infection and survival after complete system removal: An observational prospective multicenter study», Int. J. Cardiol., T. 250, ss. 146-149, Jan. 2018, doi: 10.1016 / j.ijcard.2017.07.061.

27. N. Clayton and $d r$., «Manufacture and electrical characterization of high voltage insulation for superconducting busbars - (Part 2) application techniques and electrical characteristics», Cryogenics (Guildf)., T. 98, ss. 113-124, mar. 2019, doi: 10.1016 / j.cryogenics.2019.01.003.

28. L. Wu, Q. Zhang, Z. Li, and X. Liu, "Mechanochemical syntheses of a series of bismuth oxyhalide composites to progressively enhance the visible-light responsive activities for the degradation of bisphenol-A,” Mater. Sci. Semicond. Process., T. 105, Jan. 2020, doi: 10.1016 / j.mssp.2019.104733.

29. EA Franceschini, A. Hainer, i AE Lanterna, "Niobium-based semiconductor electrodes for hydrogen evolution reaction”, Int. J. Hydrogen Energy, t. 44, vqp. 60, ss. 31940-31948, dec. 2019, doi: 10.1016 / j.ijhydene.2019.10.101.

30. [30] E.. S. B. Yu. V. Emelyanova, M. V. Morozova, Z. A. Mixaylovskaya,Impedance Spectroscopy: Theory I Application: Uchebnoe Posobie. 2017.

31. [31] L. Hui, S. Yundong, Z. Kai, P. Jingbiao, i W. Zhu, "A simplified digital positron lifetime spectrometer based on a fast digital oscilloscope", Nucl. Instruments Methods Phys. Res. Sect. A Accel. Spectrometers, Detect. Assoc. Equip., T. 625, vqp. 1, ss. 29-34, Jan. 2011, doi: 10.1016 / 
j.nima.2010.10.005.

32. [32] NG Gomaa, "The stability of MIS solar cells and evaluation by CV characteristics", Renew. Energy, t. 23, vqp. 3-4, ss. 369-374, July. 2001, doi: 10.1016 / S0960-1481 (00) 00126-9.

33. [33] M. Kaczmarek i T. Szatilo, «Reference voltage divider designed to operate with oscilloscope to enable determination of ratio error and phase displacement frequency characteristics of MV voltage transformers», Meas. J. Int. Meas. Confed., T. 68, ss. 22-31, 2015, doi: 10.1016 / j.measurement.2015.02.045. 